\title{
Vers des matériaux intelligents : les matériaux multifonctionnels
}

\section{Towards the intelligent materials word: the multifunctional materials}

\footnotetext{
工

a revue «Matériaux \& techniques » s'est de longue date intéressée au concept encore récent des matériaux «smart» ou « intelligents». En effet deux numéros hors série (décembre 2001 et juillet 2002) ont déjà abordé ce vaste sujet en rassemblant pour leurs lecteurs les contributions présentées au cours du second et du quatrième colloque bi-latéral France-Japon créé en 1997 et régulièrement consacré tous les deux ans à ce domaine.

Mais les matériaux eux-mêmes, les domaines de recherche et d'applications potentielles évoluent et un point régulier est nécessaire. La conférence «Matériaux 2006 » a été l'occasion de réunir à Dijon la majorité des acteurs nationaux. C'est à partir de cette base que nous vous présentons ici un ensemble (actualisé 2008), certes non exhaustif (il y faudrait plusieurs numéros!), mais constituant néanmoins une introduction raisonnable à un domaine bouillonnant auquel plusieurs colloques internationaux sont toujours consacrés chaque année [1-4]. . .

La notion de matériaux a évolué au cours des âges. D'abord directement issus du milieu naturel, puis élaborés de façon de plus en plus complexe, ils ont été considérés (ou souhaités) jusqu'à une période récente, comme conservant des propriétés stables conformes aux vœux des concepteurs des systèmes dans lesquels ils sont engagés. Ce n'est qu'au début des années 80 qu'est apparu - comme un rêve d'ingénieurs - le concept de matériaux et de systèmes aux propriétés évolutives dits "smart » ou «intelligents », capables de s'adapter, notamment sous l'effet d'un stimulus externe, pour remplir au mieux leur mission dans un environnement changeant.

Ce concept a été introduit dans un cadre résolument «biomimétique» où le matériau intelligent possèderait des «nerfs » représentés par un réseau de capteurs, des «muscles » simulés par des composants à propriétés ajustables et un «cerveau » modifiant grâce aux «muscles » ses caractéristiques globales.

Ce concept ambitieux du matériau «acteur de son évolution» implique naturellement la multi-fonctionnalité et s'est en fait structuré à plusieurs niveaux de difficultés croissantes :

- Matériaux simplement «sensibles » fournissant une information sur leur propre état structural et/ou leur environnement.

- Matériaux «actifs » ou «adaptables » capables de faire évoluer une de leurs caractéristiques sous l'effet d'une modification de leur environnement.

- Matériaux adaptatifs ou « intelligents » à la fois «sensibles » et «adaptables», susceptibles de réagir par eux mêmes - et dans le bon sens - à l'évolution des variables internes ou externes. Ce sont les seuls qui répondent à l'objectif initial mais contrairement aux deux premiers niveaux, les exemples bien qu' encourageants restent rares et perfectibles.
}

Ce numéro spécial n'est pas une publication de revues classique mais il propose huit contributions qui illustrent en première analyse le classement proposé plus haut.

La première attribue l'émergence des matériaux intelligents à l'ambition venue du fond des âges d'imiter le monde du vivant et suggère quelques pistes de recherche et d'applications (Matériaux Intelligents Structuraux et Biomimétisme).

Les trois articles suivants sont consacrés aux matériaux «sensibles »; aux polymères conducteurs composites (Résistivité des Élastomères Magnétorhéologiques), au suivi de l'endommagement de matériaux divers par émission acoustique et aux approches récentes du délicat problème de la ruine des structures (Identification 
de la signature Acoustique des mécanismes d'Endommagement et Prévision de la Durée de vie), ainsi qu'à la préparation des vêtements du futur (Influence des conditions Climatiques sur un Capteur d'Allongement souple, compatible Textile).

Quatre contributions traitent ensuite des matériaux «actifs » ou « commandables ». La première se consacre à l'étude de polymères innovants mécaniquement actifs ou électrochromes (Réseaux interpénétrés électrocommandables pour l'actionnement et l'électrochromisme). La seconde traite d'une application originale et en plein développement des matériaux piézoélectriques (Techniques non-linéaires semi-passives pour le Contrôle Vibratoire et la Récupération d'Énergie). Les auteurs de la troisième s'attachent à élaborer et à modéliser un matériau composite dont 'l'activité' repose sur la différence de dilatation entre les composants. Enfin le monde plein de promesses de l'haptique s'ouvre avec un matériau électrocommandable (Les Fluides magnéto-rhéologiques en tant que frein Haptique : application au rendu du toucher de piano).

Le souhait des auteurs est que la lecture de cet ensemble vous encourage à suivre l'évolution de ce vaste domaine dans l'une des manifestations - souvent récurrentes - qui y sont consacrées.

Exemples des principales manifestations récentes et/ou récurrentes :

\section{Références}

[1] ASME 2008 Conference on Smart Materials, adaptive structures \& Intelligent Systems (SMASIS), October 28-30, 2008 Turf Valley Resort, Ellicot City, USA

[2] Smart Structures and Materials, SPIE (Conférences annuelles ) (dernière édition 2008 San Diégo, USA)

[3] International Conferences on Adaptive Stuctures and Technologies (dernière édition 19e ICAST 2008) October 6-8, Ascona, Switzerland (conférence annuelle)

[4] International Workshops on Structural Health Monitoring (manifestations annuelles en alternance en Europe et aux USA (Stanford), Dernière édition EWSHM, Juillet 2008, Cracovie

P.F. Gobin et C. Chevrot

Éditeurs invités 